guidance for management, especially in children. We conducted a multi-centre retrospective service evaluation to better understand current practice.

Methods Children diagnosed with IIH from nine centres in the UK over a 3 year period were retrospectively studied. Patient demographics, clinical details at presentation and follow-up, comorbidities, investigations, and medications were recorded and analysed.

Results 105 patients (71 females) with IIH, median age 11 [IQR 5-13] were recruited. Overall, record keeping was suboptimal with no recorded weight in 33\% (43/105), height in $64 \%$ (68/105); and visual acuity in 24\% (26/105).

At presentation $42 / 62 \quad(67 \%)$ of the patients had weight $>90$ th centile, behavioural issues were reported in 15/ $63(23 \%)$ and missing school in 9/43 (20\%). Papilloedema was reported in $98 / 105(93 \%)$ at baseline and in $87 / 105$ $(82 \%)$ at 6 months. Median GA opening CSF pressure was 34 (IQR 28 to 40) $\mathrm{cm} \mathrm{H} 2 \mathrm{O}$ and non-GA was 32 (IQR 25 to 39). $1 / 105(0.9 \%)$ had ICP monitoring and $1 / 105(1 \%)$ had shunt.

$73(69 \%)$ were seen by an ophthalmologist and 48 (45\%) had no record of either orbital US scan or OCT. Only $16(15 \%)$ had MRV and/or MRA. 51 (48\%) had LP under GA, of whom only 10 (19.6\%) had CO2 monitoring recorded. 92/ 105 (87\%) received acetazolamide, however 25/92 (27\%) had no electrolytes checked during acetazolamide therapy.

Conclusions This project highlights significant variation in the way that IIH is being investigated and managed in the UK. This variation is likely to reflect the paucity of evidence, but risks over-diagnosis and inappropriate management. These results should be used to form the basis for a national consensus for the diagnosis and management of IIH in young people.

\section{G308(P) SUDDEN UNEXPECTED DEATH IN EPILEPSY (SUDEP) DURING SLEEP IN THREE 17-YEAR-OLD ADOLESCENTS WITHOUT INTELLECTUAL DISABILITY}

${ }^{1}$ A Lim, 'V Ramesh, ${ }^{2} \mathrm{~S}$ Annavarapu, ${ }^{2} \mathrm{~T}$ Polvikoski. ${ }^{1}$ Paediatric Neurology, Newcastle NHS FT, Newcastle upon Tyne, UK; ${ }^{2}$ Pathology, Newcastle NHS FT, Newcastle upon Tyne, UK

\subsection{6/archdischild-2018-rcpch.300}

Aim To report risk factor(s) that might have led to SUDEP in three similar cases.

Methods Case note reviews; post-mortem reports; interviews with bereaved guardians/parents.

Results 17-year-old male had juvenile-onset generalised epilepsy diagnosed with onset at age 15. His generalised tonic-clonic seizures (GTCS) were mainly nocturnal; controlled with Valproate and Leveteracetam. His father saw playing on his computer at $1030 \mathrm{pm}$ and was found dead in his bedroom floor in early morning. No structural brain lesion demonstrated on postmortem except incidental right temporal pole arachnoid cyst. 17-year-old male with generalised epilepsy epilepsy with febrile seizures (GEFS+) with onset at age 2. His GTCS were relatively infrequent and only during intercurrent illness and had been treated with Valproate. He had been playing videogames throughout the night and went to bed the following evening. He was found dead at $930 \mathrm{pm}$ face down in his bed. 17-year-old female had presented with first episode of GTCS secondary to previously undiagnosed autoimmune hypothyroidism at age 15. Her Hashimoto encephalopahty was treated with steroids and thyroxine. After the initial presentation, no GTCS were reported. She was on thyroxine with no anti-epileptics. She had headache the day before. She was found dead face down in her bed in the early morning.

Discussion SUDEP typically affects 1 in 4500 children. Risk factors for SUDEP are young age, male sex, early onset, GTCS, and being in bed. Sodium channel mutation epilepsies are an at risk group for SUDEP. In this review, all three patients were aged 17 years and manifested only GTCS of varying aetiology. They had no intellectual disability or known poor compliance. All had been asleep in bed prior to SUDEP. Conclusions All three adolescents were sleep-related SUDEP. Potential strategies for prevention include avoiding sleep deprivation, counselling adolescents with sodium channel epilepsies and considering nocturnal seizure detection device.

\section{G309(P) PREDICTIVE VALUE OF ELECTROENCEPHALOGRAPHY FOR DEVELOPMENTAL OUTCOME AT 6 MONTH OF AGE IN FULL TERM NEONATES WITH SEIZURES}

M Ramwani, A Singh, I Shrivastava, A Agarwal. Department of Paediatrics, Gandhi Medical College, Bhopal, India

\subsection{6/archdischild-2018-rcpch.301}

Background Electroencephalography (EEG) is an important tool to evaluate infant with symptoms refer to central nervous system. The objective of the work was to study the predictive value of EEG for developmental outcome in full term neonates with seizures at 6 month of age.

Methods This was prospective observational study at tertiary care centre, Gandhi Medical College, Bhopal. The participants were full term hospitalised neonates with documented seizures. Newborns admitted for complaints other than seizures, hypoxic ischaemic encephalopathy (HIE) stage 1, preterm and neonates born to mother on antiepileptic therapy were excluded from the study.

Results Out of total 108 registered neonates 14 expired and 10 lost to follow up. In remaining 84 cases, 36 (42.9\%) had generalised discharge, 16 (19\%) had focal and $6(7.1 \%)$ had multifocal discharge while $44(52.3 \%)$ had normal EEG patterns. HIE being the most common cause and subtle seizure being the most common type of seizures. On follow up at 6 month, all neonates with normal EEG pattern $(n=44)$ had normal developmental outcome. Out of 40 neonates with abnormal EEG discharges, 26 (65\%) had developmental delay, while 14 (35\%) had normal development for age.

Conclusions EEG could be a simple and cost effective prognostic tool where neonates presenting with seizure. Newborn with abnormal discharge should be closely monitored on DDST for early identification and early intervention.

\section{G310(P) TACKLING EPILEPSY IN A DGH: SEIZING NICE QUALITY STANDARDS AND AUDITING AGAINST CURRENT PRACTICE}

S Ali, R Piggott, S Sastry, N Gulati. General Paediatrics, The Royal Wolverhampton NHS Trust, Wolverhampton, UK

\subsection{6/archdischild-2018-rcpch.302}

Objectives To compare our clinical practice against the NICE quality standards for children's epilepsy service. 
Methods Outpatient clinic lists for consultant specialist in diagnosis and management of epilepsy were obtained for one year (April 2016 - May 2017) and outpatient letters were reviewed. All patients diagnosed with epilepsy within the last ten years were included. Patients diagnosed abroad or at another hospital were excluded.

Results A total of 119 patients were audited; 65 male and 54 female.

- Seen by specialist in the diagnosis and management of epilepsy within 2 weeks of presentation with a suspected seizure? Yes $=33, \mathrm{No}=86$

- Investigations (MRI/EEG) undertaken within 4 weeks of request? Yes $=66, \mathrm{No}=53$

- Children meeting criteria for neuroimaging have an MRI? Yes $=69, \mathrm{No}=4, \mathrm{~N} / \mathrm{A}=46$

- Agreed and comprehensive written epilepsy plan? Yes=116, $\mathrm{No}=3$

- Patients seen by epilepsy specialist nurse? No=115, N/A=4

- Agreed written emergency care plan for prolonged/repeated seizures? Yes $=14, \mathrm{No}=63, \mathrm{~N} / \mathrm{A}=42$

- Seen within 4 weeks of referral to tertiary centre if required? Yes $=6, \mathrm{No}=17, \mathrm{~N} / \mathrm{A}=96$

- Structured review with paediatric epilepsy specialist minimum annually? Yes $=107, \mathrm{No}=1, \mathrm{~N} / \mathrm{A}=11$

- Agreed transition period where care reviewed jointly by adult and paediatric services? Yes $=14, \mathrm{No}=5, \mathrm{~N} / \mathrm{A}=100$

Conclusions The results highlight our patients are having comprehensive written care plans (97\%), structured reviews annually (99\%) and are reviewed jointly for transition (74\%). Epilepsy service enhancement is needed in the time taken to be seen by the secondary care epilepsy specialist $(28 \%)$ or tertiary centre $(26 \%)$ once a referral is a made. Investigation time waits could also be improved (55\%). Quality of care could be enriched by employing an epilepsy nurse specialist.

Numerous studies have demonstrated that inadequate epilepsy care results in significant consequence. It is imperative to implement NICE quality standards to provide better quality of care to children/young people diagnosed with epilepsy.

\section{G311(P) ABSTRACT WITHDRAWN}

\section{G312(P) DMD AUDIT ABSTRACT FOR (P) PRESENTATION}

${ }^{1} \mathrm{~V}$ Sampath-Arutperumselvi, ${ }^{2} \mathrm{C}$ DeGoede. ${ }^{1}$ Paediatrics, North West Deanery, Manchester, UK; ${ }^{2}$ Paediatric Neurology, Royal Preston Hospital, Preston, UK

10.1136/archdischild-2018-rcpch.303

Objective Diagnosis and management of Duchenne muscular dystrophy: Use of published standards, to audit regional service.

Background In 2010 standards of care for diagnosis and management of Duchenne muscular dystrophy were published in Lancet Neurology. ${ }^{1,2}$ Our regional neuromuscular service was set up in 2013, largely supported and driven by these standards. We evaluated the service against these standards.

Methodology Retrospective audit looking at the management of 31 DMD patients. Data was collected by reviewing case notes over a period of 14 months.

Results All patients $(n=31,100 \%)$ had genetic testing to confirm the diagnosis and received chicken pox vaccination or had their immune status checked before starting steroids.24 patients $(80 \%)$ had attended specialist clinic appointment every 6 months. North Star Ambulatory assessment recommended every 6 months was done in 3 patients (20\%). Annual serum Vitamin D level was done in 17 patients (58\%). Annual DEXA scan for patients on chronic glucocorticoid therapy was done in 4 patients (14\%). Spinal X-ray for patients with back pain or Kypho-scoliosis was done in $77 \%$ of the patients $(\mathrm{n}=10) .30$ patients $(97 \%)$ received Vitamin D supplements and 28 patients $(93 \%)$ had echocardiograms done as per recommendation. $71 \%$ of the ambulatory patients $(n=10)$ received the recommended respiratory assessments but none of the non-ambulatory patients received all the recommended respiratory assessments. $75 \%$ of non-ambulatory patients with poor lung function $(n=3)$ received the recommended awake end tidal CO2 level measured.

Conclusions Vital areas for improvement were identified and an action plan has been formulated. Main areas for improvement included availability of the neuromuscular physiotherapist to perform 6 monthly North Star ambulatory assessment and respiratory assessment, and improving access to DEXA scan. The latter involves training radiology staff to perform scans on children. The published standards have been very helpful in the development and subsequent evaluation of the regional muscle service.

\section{REFERENCES}

1. Nushby, et al. Diagnosis and management of duchenne muscular dystrophy, part 1: Diagnosis, and pharmacological and psychological management. Lancet Neurology 2010;9:77-93.

2. Bushby, et al. Diagnosis and management of duchenne muscular dystrophy, part 2: Implementation of multidisciplinary care. Lancet Neurology 2010;9:177-189.

\section{G313(P) TIME TO ADOPT ADULT STROKE GUIDELINES FAST (FACE, ARMS, LEGS, TIME)}

S Akbar, V Tyagi. Royal Aberdeen Children's Hospital, NHS Grampian, Aberdeen, UK

\subsection{6/archdischild-2018-rcpch.304}

A previously healthy 14-year-old female was unwell for a few days with vomiting associated with mild unilateral neck pain. Whilst on the phone, she developed left sided limb weakness, slurred speech and double vision over a matter of few minutes. She was transferred to Accident and Emergency (A and E) where she had ongoing focal neurology with a left side ataxic hemiparesis and a right internuclear ophthalmoplegia. Although she was in Paediatric age-group, there was a strong suspicion of a stroke and she underwent CT imaging which showed a normal brain parenchyma. However, CT angiogram confirmed a pseudo-aneurysm in the left vertebral artery suggestive of dissection and a filling defect at the tip of the basilar artery in keeping with occlusion (likely embolus). As she was within the 4.5 hour window for thrombolysis, she was treated with Alteplase, a tissue plasminogen activator (tPA) by the on-call stroke consultant and was started on high dose Aspirin before being transferred to a tertiary Paediatric Neurology centre for ongoing care. MRI scan later confirmed bilateral pontine infarcts. At follow-up, she has a minor left sided ataxia but otherwise has recovered well. This patient presented before the publication of recent RCPCH stroke guidelines but a pragmatic decision by the $\mathrm{A}$ and $\mathrm{E}$ team ensured appropriate acute treatment. 